ternational and Austrian law, the effects of the work of the European Commission and of the Strasbourg court on the Austrian legal order and on the main direction of the rulings of Austrian courts in handling human rights questions.

The handbook's contribution to practice and research could hardly be overestimated. Although the authors at several times complain about a certain reluctance of the courts to give sufficient effect to all provisions of the Convention one should not forget that the development of human rights in many countries is still far from enabling scholars to undertake a comparable study.

Philip Kunig

\title{
Hans-H. Münkner
}

\section{Die rechtliche Regelung der Vorgenossenschaft}

Reihe Studien und Berichte Nr. 12, Institut für Kooperation in Entwicklungsländern, Marburg/Lahn, 1981, $127 \mathrm{~S}$.

Trotz zunehmender Kritik und zahlreicher Fehlschläge staatlicher Genossenschaftsgründungen in Ländern der Dritten Welt gelten die Genossenschaften in den Entwicklungsländern häufig noch als die Organisationsform, "die den wirtschaftlich und sozial schwächer gestellten Bevölkerungsteilen eine Möglichkeit eröffnen soll, durch Zusammenschluß und organisiertes Zusammenwirken aus eigener Kraft eine stärkere wirtschaftliche und soziale Stellung zu erlangen" (S. 73). Im Gegensatz zu lokalen Selbsthilfegruppen werden sie von der staatlichen Administration daher als örtliche Entwicklungszellen betrachtet, die als Anknüpfungspunkte staatlicher Entwicklungsplanung dienen. Dennoch sind die vielfältigen Probleme der "Genossenschaft im Gründungsstadium", die der Verfasser mit dem Begriff der "Vorgenossenschaft" umschreibt, unübersehbar. Als die gravierendsten sind hier zu nennen: der Mangel an erfahrenen lokalen Gründern sowie das mangelnde Eigenkapital der (Gründungs-)Mitglieder als Voraussetzung einer selbständig und effektiv arbeitenden örtlichen Wirtschaftsorganisation; aber auch die Ausrichtung der von staatlichen Promotoren gegründeten Genossenschaften auf die staatlichen Entwicklungsziele anstelle einer Orientierung auf die Ziele der Mitglieder als Träger der Genossenschaften, was besonders in traditionell geprägten Gebieten zu besonderen Organisationsproblemen führte.

Der Schaffung eines rechtlichen Rahmens zur Bewältigung dieser Probleme der Vorgenossenschaft gilt die vorliegende rechtsvergleichende Schrift. Das besondere Interesse des Verfassers ist dabei darauf gerichtet, die "entscheidende Frage nach dem Zugang der Entwicklungsplaner und -promotoren zu den in Armut lebenden ländlichen Bevölkerungsgruppen einer Lösung näherzubringen" (S. 5), wobei der Blick auf die Gewährleistung einer situationsgerechten eigenen Interessenartikulation und Durchsetzung der jeweiligen Bevölkerungsgruppen, insbesondere der ländlichen Kleinproduzenten, vor einer Orientierung auf nationale Entwicklungsziele gerichtet ist. Diese organisatorische Selb- 
ständigkeit der Vorgenossenschaft will der Verfasser in seinen Vorschlägen zur rechtlichen Regelung der Vorgenossenschaft durch eine Autonomie der Gründungsmitglieder bei der Ziel- und Zwecksetzung der Genossenschaftsgründung gewahrt wissen. Er stützt sich hier auf die Erfahrungen lokaler Selbsthilfegruppen und versteht die Genossenschaft im Gründungsstadium als Lernphase genossenschaftlichen Handelns und genossenschaftlicher Organisation. Entsprechend offen ist das Gründungsverfahren unter Verzicht auf detaillierte Regelungen sowie die Wahrnehmung von Mitgliedsrechten zu gestalten. In dieser Lernphase sieht er aber auch Anknüpfungspunkte für die Tätigkeit staatlicher Entwicklungspromotoren und nationale Entwicklungskonzepte, die jedoch durch dezentrale Förderungsorganisationen und flexible Richtlinien den örtlichen Gegebenheiten angepaßt sein müssen. Einem zu großen Einfluß der Entwicklungspromotoren auf die Vorgenossenschaft soll durch die Beschränkung ihrer Aufgaben auf die Beratung und Anleitung und die Untersagung einer Ubertragung von Exekutivfunktionen ein Riegel vorgeschoben werden. Weitere Regelungsvorschläge gelten: dem genossenschaftlichen Willensbildungsprozeß; der Finanzierung, die von dem Prinzip der Eigenfinanzierung geleitet ist; der Haftung und dem Gläubigerschutz sowie der Umwandlung der Vorgenossenschaft in eine Genossenschaft.

Die Untersuchung zeigt die in Entwicklungsländern bestehenden Schwierigkeiten einer allumfassenden nationalen Gesetzgebung, die vor allem auf traditionelle - nicht notwendig rückständige - Widerstände stößt. Entsprechend of fen sind rechtliche Regelungen zu halten, um einer Entwicklung "von unten", die auch für die Akkulturation rechtlicher Normen und die Verhinderung eines rechtlichen Dualismus Bedeutung hat, einen gewissen Raum zu geben. Dies wird vom Verfasser nicht nur im Vergleich am Beispiel der Genossenschaftsgründung in Obervolta, sondern auch in seinen Ausführungen zu den Gründungsvoraussetzungen, dem Gründungsverfahren, der Gründungsprüfung und der Eintragung ins Genossenschaftsregister hervorgehoben. Aus ihnen sind die genannten Regelungsvorschläge erwachsen. Ihre an eine Checkliste erinnerende Kürze ist auf diesem Hintergrund zu sehen. So mag der eine exakte Regelung erwartende Leser zwar unbefriedigt sein, offene Fragen lassen sich jedoch leicht durch einen Rückgriff auf die vom Verfasser zum Vergleich herangezogenen Regelungen aus Niger, Obervolta, Kenya, Tanzania, Uganda, dem Senegal und der Elfenbeinküste sowie den Philippinen und den darauf beruhenden Ausführungen zu dem gesamten Gründungsstadium und -verfahren beantworten. Erschwerend wirkt hier jedoch die häufig fehlende, wegen des institutionellen Zusammenhangs oft nicht mögliche begriffliche Trennung zwischen Vorgenossenschaft und Genossenschaftsgründung.

Unverkennbar ist die Ausrichtung der Untersuchung auf Afrika. Die profunden Kenntnisse des Verfassers im afrikanischen Genossenschaftsrecht und -wesen sowie die Einbeziehung der Samahang Nayon der Philippinen bieten jedoch genügend Ansätze, die eine Ubertragung der Erkenntnisse auf andere Regionen erlauben. Die außerordentlich praxisorientierte Untersuchung dürfte daher auch für die Genossenschaftsberatung in anderen Regionen von großem Nutzen sein.

Ulrich Werwigk 